Research Article

\title{
Fracture Toughness Reliability in Polycarbonate: Notch Sharpening Effects
}

\author{
A. Salazar, ${ }^{1}$ J. Rodríguez, ${ }^{1}$ and A. B. Martínez ${ }^{2}$ \\ ${ }^{1}$ DIMME, Departamento de Tecnología Mecánica, Escuela Superior de Ciencias Experimentales y Tecnología, \\ Universidad Rey Juan Carlos, C/Tulipán, s/n, Móstoles, 28933 Madrid, Spain \\ ${ }^{2}$ Centre Català del Plàstic, Universitat Politècnica de Catalunya, C/Colom 144, Terrassa, 08222 Barcelona, Spain
}

Correspondence should be addressed to A. Salazar; alicia.salazar@urjc.es

Received 10 October 2013; Accepted 27 November 2013

Academic Editors: W. Cantwell and A. Kajbafvala

Copyright (C) 2013 A. Salazar et al. This is an open access article distributed under the Creative Commons Attribution License, which permits unrestricted use, distribution, and reproduction in any medium, provided the original work is properly cited.

\begin{abstract}
The effect of the notch sharpening on the fracture toughness obtained under linear elastic fracture mechanics has been analyzed in an amorphous polycarbonate. The samples for fracture characterization were sharpened via the traditional contact steel razor blade technique and the noncontact femtosecond laser ablation technique. The values of the fracture toughness of the specimens sharpened through femtosecond laser ablation were lower than those measured on samples sharpened using a steel razor blade. Moreover, the former was in plane strain state, but the latter did not verify the size criterion. The damage produced ahead of the crack tip through plastic deformation in the steel razor blade sharpened samples over the lack of damage in the femtolaser sharpened specimens explains the differences in the fracture toughness. It has been proven that there is a relationship between the plastic deformation at the crack front and the stress state. This has been assessed through the application of a fracture criterion for dissipative systems with small scale yielding.
\end{abstract}

\section{Introduction}

The influence of crack sharpness on the fracture toughness values of polymeric materials has recently caught the attention of the scientific community. Several works have appeared in the literature highlighting the role of the notch sharpening technique on the fracture parameters of either semicrystalline heterogeneous polymeric materials such as ethylene-propylene block copolymers [1-3] or amorphous polymers such as polycarbonate [4]. In all these studies, the values of the fracture toughness obtained using different approaches were determined from samples sharpened via two procedures: a noncontact technique based on the femtosecond laser ablation (femtolaser) and the traditional contact technique using a razor blade. Interestingly, both sharpening techniques resulted in similar initial crack tip radii for these polymers, and the fracture toughness determined from the samples sharpened via the femtolaser showed lower values than those obtained from the specimens sharpened via the traditional razor blade technique independently of the material under study. These differences reached in the copolymer values up to $11 \%, 75 \%$, and $90 \%$ when the crack growth initiation parameters were computed under linear elastic fracture mechanics (LEFM), elastic-plastic fracture mechanics (EPFM) and Post-Yielding Fracture Mechanics (PYFM) conditions, respectively, [3] and radical changes in the J-R curves obtained under EPFM approximation in the polycarbonate [4]. The reason is that the femtosecond pulsed laser ablation is characterized by very rapid creation of vapor and plasma phases, negligible heat conduction, and the absence of liquid phase preventing melting and thermal deformations of the surrounding area [5]. However, the specimens sharpened using the razor blade always showed damage ahead of the crack tip in the form of plastic deformation which seems to be the reason of the higher fracture toughness values. Moreover, the abrupt differences between the fracture toughness values, especially under EPFM [3, 4] and PYFM [3] obtained from samples sharpened via different techniques 
seem to indicate that there is a relationship between the notching procedure and the stress.

In the light of the previous results, the aim of the present work is to analyze more deeply the possible relationship between sharpening technique and the stress state developed ahead of the crack tip during the fracture process. For that, the fracture toughness under LEFM will be determined in an amorphous polymer such as polycarbonate. The fracture specimens will be sharpened via the traditional razor blade procedure and the femtolaser technique. The results will be analyzed with the help of the fracture criterion for dissipative systems with small-scale yielding behavior occurring under LEFM conditions [6].

\section{Materials and Methods}

The material under study is an amorphous polycarbonate sheet, PC, supplied by Nudec. The glass transition temperature was $163^{\circ} \mathrm{C}$. Five tensile specimens were tested at $23^{\circ} \mathrm{C}$ and at a crosshead speed of $1 \mathrm{~mm} / \mathrm{min}$ following the ISO527 standard guidelines. The corresponding mean values together with the standard deviations of the Young's modulus and the yield stress were $E=1.94 \pm 0.02 \mathrm{GPa}$ and $\sigma_{Y}=60.3 \pm 0.1 \mathrm{MPa}$, respectively.

At least, five valid fracture tests were carried out on single edge notch bend specimens with $5 \times 10 \times 44 \mathrm{~mm}$ in size. An initial straight-through slot with a length to width ratio of 0.5 and terminating in a $\mathrm{V}$-notch with $0.15 \mathrm{~mm}$ in root radius was machined. The notch was sharpened via two procedures as follows.

S-Type. It consists of sliding a fresh steel razor blade across the root of the $\mathrm{V}$-notch following the guidelines described by ESIS [7].

F-Type. The sharpening is produced through Femtolaser [5], using a commercial Ti: sapphire oscillator (Tsunami, Spectra Physics) plus a regenerative amplifier system (Spitfire, Spectra Physics) based on chirped pulse amplification (CPA) technique. Linearly polarized 120 -fs pulses at $395 \mathrm{~nm}$ with a repetition rate of $1 \mathrm{kHz}$ were produced. The scanning speed was $130 \mu \mathrm{m} / \mathrm{s}$. Four passes were carried out with a pulse energy of $0.008 \mathrm{~mJ}$. The sharp length inserted by the femtolaser was $500 \mu \mathrm{m}$.

The total initial crack depth, $a_{0}$, to width ratio after sharpening was 0.55 .

The morphology and dimensions of the crack tip after sharpening and the area behind it of the nontested specimens as well as the morphology of the fracture surfaces were analyzed via scanning electron microscopy (Hitachi S-3400N).

The fracture tests were carried out at room temperature and under displacement control at a cross-head speed of $1 \mathrm{~mm} / \mathrm{min}$, using a three-point bending fixture with a loading span to width ratio of 4 mounted on an electromechanical testing machine (MTS Alliance RF/100). The applied load was measured with a load cell of $\pm 5 \mathrm{kN}$. The mechanical response of the $\mathrm{PC}$ at room temperature is linear and elastic till fracture, so the ESIS TC4 protocol entitled " $K_{C}$ and $G_{C}$ at
TABLE 1: Fracture toughness values, $K_{\mathrm{IC}}$ and $G_{\mathrm{IC}}$, of polycarbonate.

\begin{tabular}{lcc}
\hline & $K_{\mathrm{IC}}\left(\mathrm{MPa} \cdot \mathrm{m}^{1 / 2}\right)$ & $G_{\mathrm{IC}}\left(\mathrm{kJ} / \mathrm{m}^{2}\right)$ \\
\hline S-Type & $3.0 \pm 0.2$ & $4.4 \pm 0.4$ \\
F-Type & $4.1 \pm 0.3$ & $7.7 \pm 1.1$ \\
\hline
\end{tabular}

slow speeds for polymers" [7] was followed to determine the fracture toughness, $K_{\mathrm{IC}}$. The size requirement for plain strain $K_{\mathrm{IC}}$ is given by

$$
B, a, W-a>2.5\left(\frac{K_{\mathrm{IC}}}{\sigma_{Y}}\right)^{2},
$$

where $B$ is the thickness and $W$ is the width of the specimen.

\section{Results}

Table 1 shows the fracture toughness, $K_{\mathrm{IC}}$, and the critical energy release rate, $G_{\mathrm{IC}}$, of S-Type and F-Type specimens. Firstly, both the $K_{\mathrm{IC}}$ and $G_{\mathrm{IC}}$ values of the F-Type samples are lower than those from the S-Type specimens, reaching differences of up to $37 \%$. It is worth noting that the fracture toughness values obtained from S-Type specimens are analogous to those found in the literature [6]. Secondly and attending to (1), the values of the F-Type samples are in plane strain state but those determined from the S-Type specimens do not verify the size criterion.

The inspection of the crack tip front of the nontested specimens after sharpening revealed the reason of these differences. Figures 1 and 2 display the appearance of the crack tip and the zone ahead of it for F-Type and S-Type sharpened samples, respectively. The crack tip has been marked with an arrow. Both sharpening techniques provided similar crack tip radii; more accurately, the F-Type specimens presented crack tip radii of $1 \mu \mathrm{m}$ while the $\mathrm{S}$-Type samples of $1.5 \mu \mathrm{m}$. However, there is a damage area ahead of the crack tip in the S-type samples (outlined with dots) (Figure 2(a)) which was not observed in the F-Type samples (Figure 1). This zone ahead of the crack tip in the S-Type sharpened samples revealed the presence of plastic deformation caused during the notch sharpening (Figure 2(b)). Quantitative analysis of the damage area at the surface of the virgin specimens provided zones ahead of the crack tip with lengths of $100 \pm 20 \mu \mathrm{m}$. The stresses generated when sliding along the notch root with the steel blade may have surpassed the yield stress locally, giving rise to local plastic deformation. In contrast with the S-Type sharpened samples, the F-Type sharpened specimens were characterized by complete absence of damage (Figure 1(b)).

\section{Discussion}

Attending to the previous results, there seems to be a relationship between the stress state and the influence of the notch sharpening technique. The plastic zone size to specimen thickness ratio is very much associated with the stress state. In order to assess this, the fracture criterion for dissipative systems with small scale yielding was applied [6]. As first approximation, the plastic deformation developed 


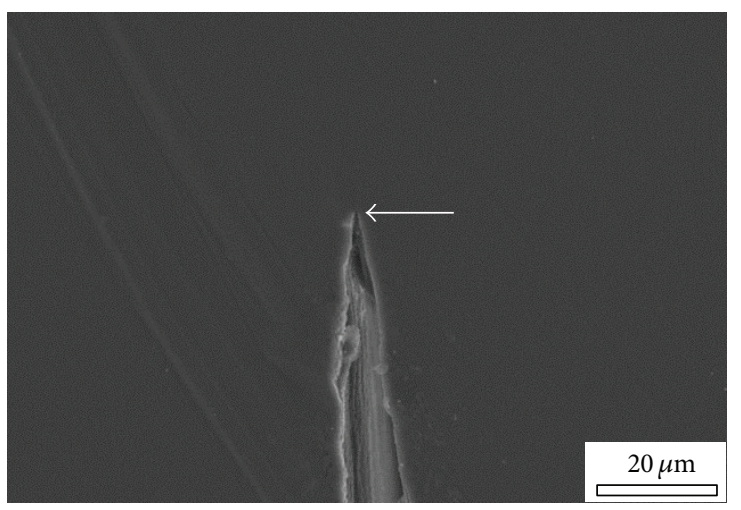

(a)

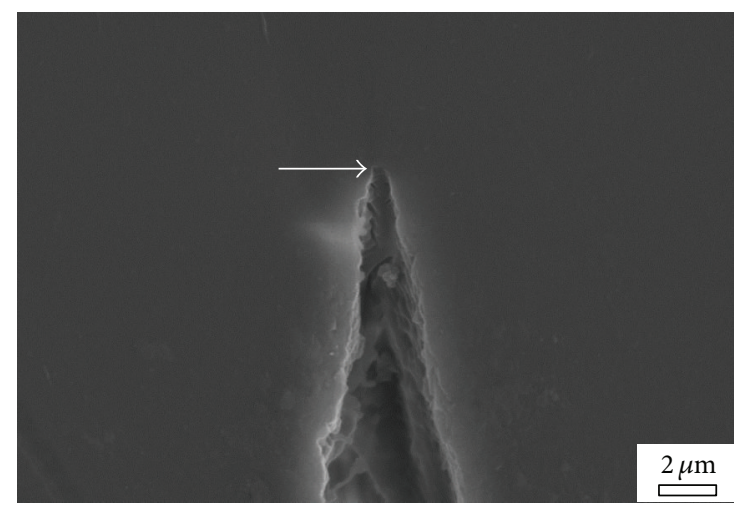

(b)

FIGURE 1: Micrographs of polycarbonate obtained via scanning electron microscopy of the crack tip front of the nontested F-Type sharpened specimens: (a) panoramic view where the arrow points out the end of the crack tip and (b) detail of the crack front.

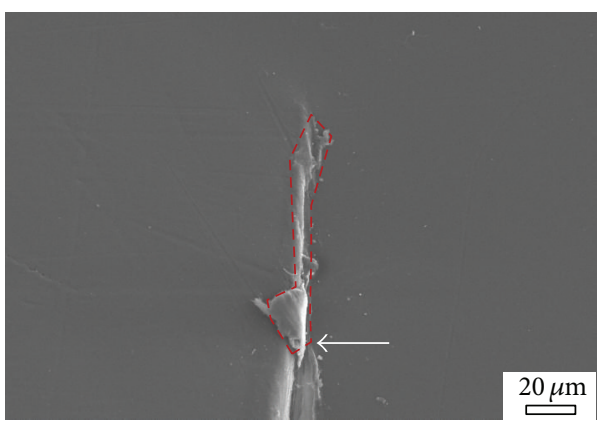

(a)

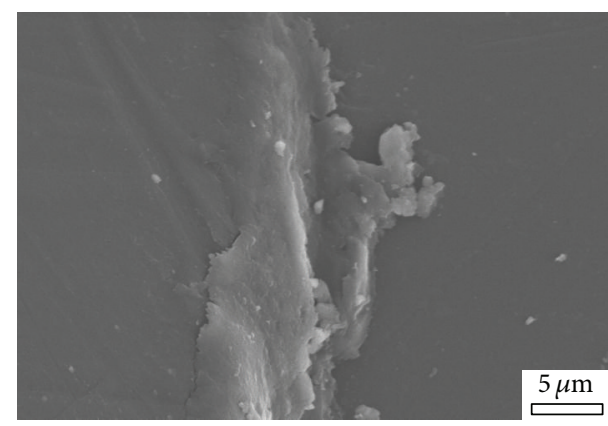

(b)

FIGURE 2: Micrographs of polycarbonate obtained via scanning electron microscopy of the crack tip front of the nontested S-Type sharpened specimens: (a) panoramic view where the arrow points out the end of the crack tip and the damage area has been outlined and (b) detail of the crack front.

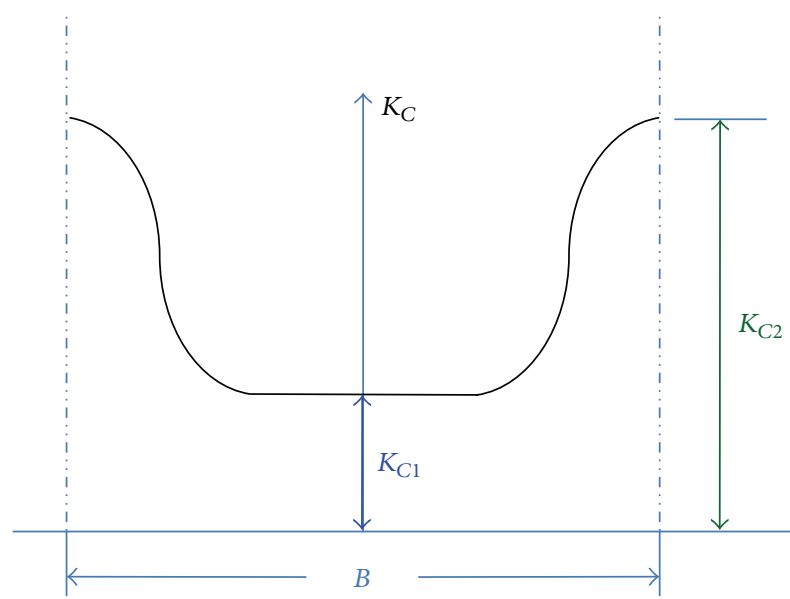

FIGURE 3: $K_{C}$ distribution across the crack front [6].

ahead of the crack tip just prior to the fracture process gives rise to a $K_{C}$ distribution across the crack front as shown in Figure 3.

Assuming a bimodal distribution with plane stress $K_{C 2}$ fracture toughness over the surface zones and plane strain
$K_{C 1}$ fracture toughness in the centre area, the measured fracture toughness $K_{C}^{\prime}$ can be attained through the expression [6]

$$
K_{C}^{\prime}=K_{C 1}+\frac{K_{C 2}^{2}}{\pi B \sigma_{Y}^{2}}\left(K_{C 2}-K_{C 1}\right)
$$

or in terms of energy

$$
\sqrt{G_{C}^{\prime}}=\sqrt{G_{C 1}}+\frac{G_{C 2} E}{\pi B \sigma_{Y}^{2}}\left(\sqrt{G_{C 2}}-\sqrt{G_{C 1}}\right) .
$$

Identifying $K_{C 1}$ and $G_{C 1}$ with the fracture toughness and fracture resistance determined from the F-Type samples and $K_{C}^{\prime}$ and $G_{C}^{\prime}$ with those obtained from the S-Type specimens, the plane stress fracture values, $K_{C 2}$ and $G_{C 2}$, can be computed via (2) and (3) (Table 2). Interestingly, the plane stress fracture resistance, $G_{C 2}$, is analogous to the fracture values obtained in PC films through the essential work of fracture methodology $[8,9]$. 
TABLE 2: Plain strain fracture toughness obtained from F-Type samples, $K_{C 1}$ and $G_{C 1}$; fracture toughness obtained from S-Type specimens, $K_{C}^{\prime}$ and $G_{C}^{\prime}$; and plane stress fracture toughness, $K_{C 2}$ and $G_{C 2}$.

\begin{tabular}{lcc}
\hline & $K_{\mathrm{IC}}\left(\mathrm{MPa} \cdot \mathrm{m}^{1 / 2}\right)$ & $G_{\mathrm{IC}}\left(\mathrm{kJ} / \mathrm{m}^{2}\right)$ \\
\hline Plane strain & $K_{\mathrm{C} 1}=3.0 \pm 0.2$ & $G_{C 1}=4.4 \pm 0.4$ \\
F-Type & $K_{C}^{\prime}=4.1 \pm 0.3$ & $G_{C}^{\prime}=7.7 \pm 1.1$ \\
S-Type & $K_{\mathrm{C} 2}=5.3$ & $G_{C 2}=14.3$ \\
\hline
\end{tabular}

\section{Conclusions}

The fracture toughness of the polycarbonate is strongly dependent on the sharpening notch technique. The specimens sharpened using the traditional razor blade technique presented damage in the form of plastic deformation ahead of the crack tip which led to higher values of the fracture toughness in comparison with those obtained from specimens sharpened via the noncontact femtolaser procedure. Moreover, the fracture toughness values determined from the femolaser were in plane strain state but not the razor blade sharpened samples. This evidences that there is a relationship between the plastic deformation generated during the sharpening procedure and the stress state.

\section{Acknowledgments}

The authors are indebted to Ministerio de Economía y Competitividad of Spain for their financial support through the project MAT2012-37762 and to NUDEC S.A. for the supply of the PC sheets.

\section{References}

[1] A. Salazar, J. Rodríguez, A. Segovia, and A. B. Martínez, "Influence of the notch sharpening technique on the fracture toughness of bulk ethylene-propylene block copolymers," Polymer Testing, vol. 29, no. 1, pp. 49-59, 2010.

[2] A. Salazar, A. Segovia, A. B. Martínez, and J. Rodríguez, "The role of notching damage on the fracture parameters of ethylenepropylene block copolymers," Polymer Testing, vol. 29, no. 7, pp. 824-831, 2010.

[3] A. Salazar, J. Rodríguez, A. Segovia, and A. B. Martínez, "Relevance of the femtolaser notch sharpening to the fracture of ethylene-propylene block copolymers," European Polymer Journal, vol. 46, no. 9, pp. 1896-1907, 2010.

[4] A. Salazar, J. Rodríguez, and A. B. Martínez, "The role of notch sharpening on the J-fracture toughness of thermoplastic polymers," Engineering Fracture Mechanics, vol. 101, pp. 10-22, 2013.

[5] P. Moreno, C. Méndez, A. García, I. Arias, and L. Roso, "Femtosecond laser ablation of carbon reinforced polymers," Applied Surface Science, vol. 252, no. 12, pp. 4110-4119, 2006.

[6] J. G. Williams, Fracture Mechanics of Polymers, Ellis Horwood, Chichester, UK, 1984.

[7] D. R. Moore, A. Pavan, and J. G. Williams, Fracture Mechanics Testing Methods for Polymers, Adhesives and Composites, Elsevier, Amsterdam, The Netherlands, 2000.
[8] A. Al-Jabareen, S. Illescas, M. Sánchez-Soto, and O. O. Santana, "Trabajo esencial de fractura de láminas de policarbonato modificado mediante extrusión reactiva con PET," Anales de Mecánica de la Fractura, vol. 25, pp. 275-280, 2005.

[9] T. Bárány, T. Czigány, and J. Karger-Kocsis, "Application of the essential work of fracture (EWF) concept for polymers, related blends and composites: a review," Progress in Polymer Science, vol. 35, no. 10, pp. 1257-1287, 2010. 

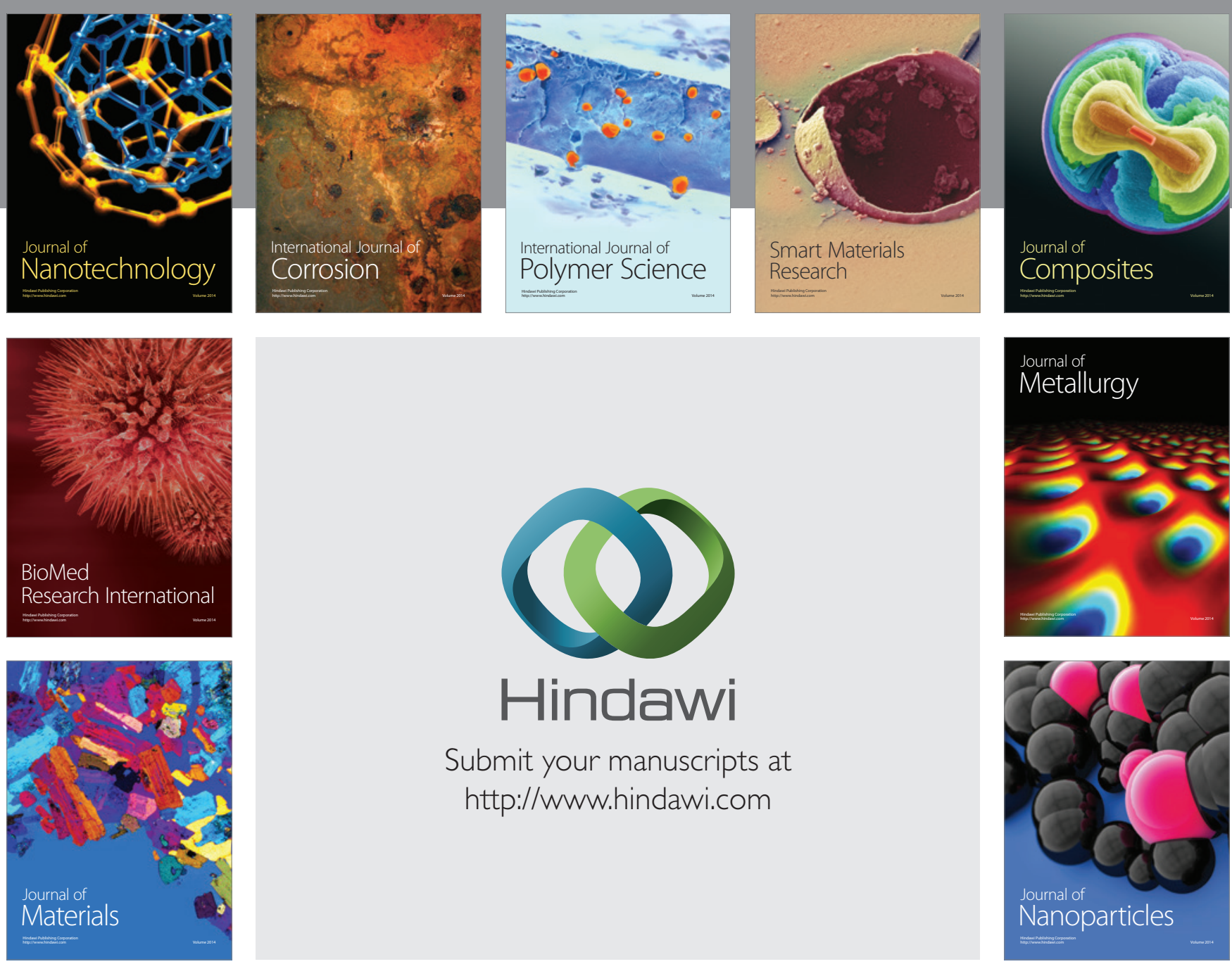

Submit your manuscripts at http://www.hindawi.com
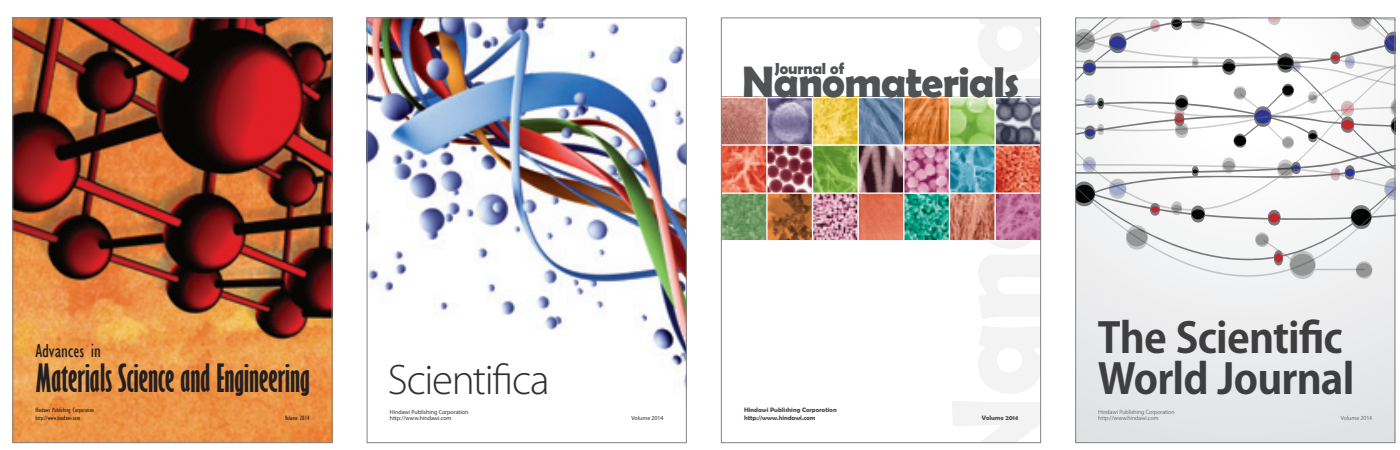

\section{The Scientific World Journal}
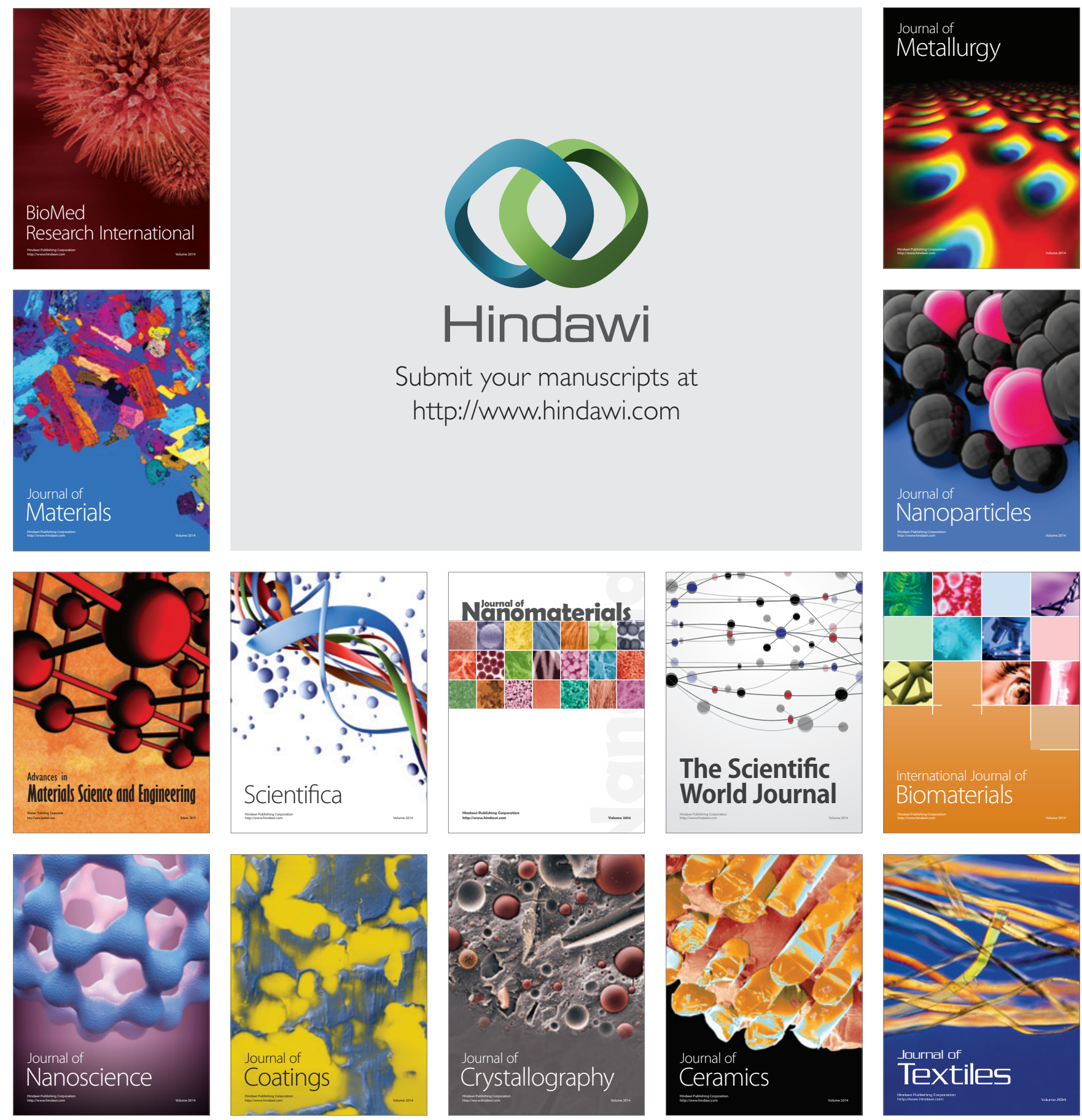